\title{
Enseñar E/LE a la primera generación de nativos digitales ${ }^{1}$
}

\author{
ALICIA HERNÁNDEZ LIECHTI \\ Lycée Français Marie Curie de Zurich \\ ahernandez@1fz.educanet2.ch
}

\begin{abstract}
Resumen: Este artículo trata de la primera generación de verdaderos nativos digitales en Europa, nacidos en torno al año 2000 y que en la actualidad asisten a los institutos de secundaria. Son diferentes a los de generaciones anteriores debido sobre todo al impacto que la tecnología ha tenido en sus vidas, pero la mayoría sigue sistemas educativos desfasados, diseñados para alumnos del siglo pasado. Muchos profesores intentan adaptarse a la nueva situación incorporando las TIC en sus cursos. La autora analiza el proceso de creación de un telediario, actividad realizada en el marco de un proyecto piloto de uso de los Ipad en los cursos de E/LE. Este proyecto obtuvo el Primer Premio del concurso a la Mejor práctica docente de E/LE basada en las TIC convocado por la Editorial Difusión/Prolinter en 2017, con la colaboración del Instituto Cervantes y el patrocinio de SIELE y FEDELE.
\end{abstract}

Palabras clave: E/LE con Ipads, nativos digitales, enfoque por proyectos, tarea final.

\begin{abstract}
This article concerns the first generation of true digital natives in Europe, children born in 2000, who, today, attend colleges or secondary schools. They are different to the previous generations due to the important impact of technology on their lives. However, they are compelled to attend schools with an outdated educational system designed for the older generations. Some teachers try to adapt themselves to the new situation by incorporating the ICT. The Spanish teacher at the LFZ therefore analyzes in this article the elaboration process of a newscast, whilst using the school iPads with her students of second year of Spanish. This video was presented at a wellknown contest amongst language teachers, where the best teaching practice of Spanish as a foreign language based on the use of ICT is selected, and won the first prize in March 2017.
\end{abstract}

Keywords: S/FL, digital natives, S/FL with Ipads, Task-based language learning.

\footnotetext{
${ }^{1}$ Realización de un Telediario. Todo con el Ipad. Primer Premio del concurso de la Editorial Difusión Prolinter a la mejor práctica docente de E/LE basada en las TIC.
} 


\section{Introducción}

El inicio del siglo XXI coincide, al menos en Europa, con el surgimiento y rápido desarrollo de la era digital, que se produce una década más tarde que en EEUU. Por lo tanto, aquellos niños nacidos en torno al año 2000 son la primera generación de verdaderos nativos digitales, es decir, estos que están hoy en nuestros institutos de secundaria y que van a invadir próximamente nuestras universidades.

Desde que Mark Prensky (2001: 1) acuñara el término nativo o inmigrante digital, la discusión sobre el tema en las salas y foros de profesores no ha cesado. Todos comentábamos, en general en términos críticos, lo que nos esperaba, muchos hacíamos cursillos de formación y casi todos comenzamos a incorporar tímidamente algunas prácticas relacionadas con las TIC. ${ }^{2}$ Tan ocupados estábamos con nuestra labor cotidiana de cumplir el currículum y la gran carga administrativa y de reuniones que sufrimos en los últimos tiempos, que casi no nos hemos dado cuenta de que los verdaderos nativos digitales ya están aquí, ocupando nuestras clases, dormitando la mayoría de las veces o puesta su atención en off, criticando a muchos de los profesores que, incluso los más experimentados, asisten impotentes a un hecho indiscutible: que estos jóvenes son diferentes y que no responden igual a los métodos y actividades que tan bien nos funcionaban hace solo un par de años.

Muchos se conforman diciendo que los alumnos ahora son más incultos, que no leen, que no saben escribir, que son indisciplinados o simplemente vagos. Ignoran que la mayoría de ellos después de la escuela se conecta, posiblemente para jugar, pero también para leer, ver películas y series -con frecuencia en otros idiomas- para contactar con gente y comentar temas afines -por escrito-, escuchar o hacer música. Todo ello con unas habilidades extremas adquiridas por ellos mismos, con las que aprenden lo que de verdad les interesa, lo que les apasiona.

\section{La escuela del siglo XX versus alumnado del siglo XXI}

Sin embargo, la escuela es otra cosa. Allí están obligados a escuchar, inmóviles, durante horas. La realidad es, que pese a los tibios intentos de mejora, en muchas de nuestras escuelas y universidades rigen todavía métodos desfasados: predomina la clase magistral y la frontalidad. Se siguen manuales, la mayoría anticuados. Existe la coartada de hacer concesiones a la modernidad por usar un ordenador, en general obsoleto, un beamer para proyectar apuntes, una presentación power point hecha hace años o un documental de Youtube. Pero con todo ello, no nos estamos adaptando a los jóvenes de hoy, que están muchísimo más avanzados en términos de tecnología y viven en un mundo ultraconectado y altamente tecnificado, un mundo en el que, de eso no hay duda, la escuela en general se ha quedado desconectada. ${ }^{3}$

\footnotetext{
${ }^{2}$ Según las encuestas realizadas por la OCDE, el promedio del conjunto de países participantes muestra que los profesores no hacen un uso sistemático de las TIC, aunque el 40\% declara que utiliza las TIC con frecuencia. Regards sur l'éducation (2015: 560)

${ }^{3}$ En la Cumbre Mundial para la Innovación en Educación (Wise, en inglés), un think tank formado por 15.000 expertos entre los que participó Noam Chomsky, se debatió sobre cómo será la escuela en 2030. Sus conclusiones fueron que desaparecerá la clase magistral; el profesor se convertirá en guía del alumno; el aprendizaje será personalizado, permanente y más caro: primarán las habilidades frente al saber académico, Internet será la principal fuente y el inglés, la lengua mayoritaria.
} 
El progreso en todos los ámbitos (sin entrar aquí en el tema de si los aportes han sido positivos o negativos para la humanidad) ha venido siempre de la mano de innovaciones técnicas o tecnológicas. Pensemos en la industria, en la medicina o en las ciencias. Novedad de nuestros tiempos es que la tecnología ha invadido el ámbito privado. Por otro lado, es evidente el enorme impacto que esta, en concreto internet, ha tenido en el mundo del conocimiento. ¿Por qué entonces la escuela y gran parte del profesorado continúa resistiéndose a incorporar la tecnología en sus clases? Los alumnos son diferentes y nuestra obligación como educadores y profesores es adaptarnos a ellos. Si en la preparación de nuestros cursos tenemos en cuenta numerosos factores como edad, origen, lengua materna, efectivos de clase, educación previa o necesidades especiales, por mencionar solo unos cuantos, habrá que considerar igualmente las características y peculiaridades de esta nueva generación.

\section{El papel del profesor y la formación}

El profesor crítico se entrega a la formación, camino inevitable para adquirir las destrezas necesarias. Pero la formación en tecnología tiene muchos inconvenientes. El mayor es el del curso en sí al que asisten: con frecuencia los formadores no han experimentado bien las técnicas que intentan enseñar. Por poner solo un ejemplo. En los últimos años se habla mucho de trabajo colaborativo, de flipped classroom o clase invertida o gamificación. Cuando los formadores, normalmente en talleres, intentan mostrar estas formas de trabajo en la práctica, suele producirse una agitación enorme y casi nunca funciona, por muchas razones, pero sobre todo porque los mismos formadores no han experimentado lo suficiente. Las limitaciones técnicas suponen otro problema evidente: la conexión todavía es muy inestable en las escuelas y centros de formación o el material muy antiguo. Así que los cursos formativos suelen ser muy frustrantes. Por no hablar de la vuelta a tu propio entorno, con pocos ordenadores para muchos alumnos, los mismos problemas de conexión y de material obsoleto.

\section{EI modelo BYOD}

Las iniciativas BYOD (bring your own device) ${ }^{4}$ o BYOT (Bring Your Own Technology), extendidas en el mundo profesional y que han comenzado a utilizarse en algunas escuelas, aportan un cierto alivio a la penuria informática de los centros. En la Guia para directores y docentes (2016: 7) del Instituto Nacional de Tecnologías Educativas y de Formación del Profesorado se explica así:

BYOD permite a los trabajadores y estudiantes llevar sus propios dispositivos móviles (ordenadores portátiles, netbooks, tabletas, teléfonos inteligentes, etc.) a sus centros de trabajo y centros educativos, y conectarlos a una red wi-fi, para acceder a aplicaciones y servicios corporativos e institucionales, y educativos, en el caso de los estudiantes.

INTEF (2016: 7)

Es un hecho que el material informático se queda obsoleto en pocos años. Antes la gente disponía de un buen ordenador en la empresa o en la escuela y ninguno o uno barato en casa. Sin embargo, debido a los avances en la tecnología de consumo, esta

\footnotetext{
${ }^{4}$ Traducción al español : "Trae tu propio dispositivo"
} 
tendencia se ha invertido, por lo que hoy en día es más normal que los usuarios dispongan de tecnología más eficaz, avanzada y productiva que la que pone a su disposición la propia empresa o en nuestro caso, la escuela. Concretamente los adolescentes desde hace solo dos o tres años poseen, prácticamente todos, un smartphone. Es decir, cada alumno tiene en su bolsillo una herramienta poderosísima que permite búsquedas, consultar diccionarios, usar la cámara y utilizar innumerables aplicaciones muy beneficiosas. No obstante, en muchas ocasiones encontramos que el autorizar a los alumnos a utilizar sus móviles va en contra del reglamento escolar. Además, los profesores que lo permiten son mal vistos y criticados por sus colegas. Sin embargo, BYOD permite incorporar algunas actividades en las clases sin la excusa de no disponer del material necesario.

\section{El alumno nativo digital en el siglo XXI}

Sin querer caer en estereotipos, el joven nativo digital es, en inquietudes, formas y estilos de vida, el más globalizado y universal. Gracias o a pesar del teléfono inteligente y la conectividad, los jóvenes españoles, suizos, japoneses o norteamericanos se parecen mucho, ya que todos dominan el mismo lenguaje: el digital.

¿Cómo se comportan estos jóvenes en clase? Los considerados buenos estudiantes son con frecuencia los dóciles, conformistas y ambiciosos, que se sienten cómodos en un entorno controlado donde se valora el esfuerzo dirigido a obtener buenos resultados. Estos solían ser también los exitosos, los que alcanzan las notas para obtener una plaza en universidades cada vez más restrictivas por el numerus clausus y otras medidas de selección. En general, se puede decir que el sistema educativo imperante está diseñado para estudiantes modelo de este tipo. Estudiantes que cada vez son menos frecuentes y que además se encuentran con que, cuando terminan sus carreras, a veces con expedientes brillantes, no consiguen un trabajo y se ven fuera del mercado laboral por falta de experiencia práctica y profesional, mal conocimiento de idiomas (pese a haberlos estudiado durante años) $\mathrm{u}$ otras múltiples carencias en otras competencias ${ }^{5}$ (Tejedor, 2013).

No obstante, cada vez son más los alumnos críticos, acostumbrados a decir su opinión en voz alta, inteligentes y muy creativos, resistentes a métodos que consideran aburridos y anticuados, como el dictado, el monólogo, los ejercicios de gramática, escuchar y tomar apuntes o copiar, es decir lo que, a día de hoy, en 2017, sigue siendo rutina en nuestras aulas europeas. Son indisciplinados, se levantan y hablan sin autorización y sufren medidas disciplinarias, cuya frecuencia ha aumentado en los últimos tiempos, debido a la impotencia de los profesores, conserjes y autoridades escolares ante tanto ruido, indisciplina, mobbing, bullying y falta de atención en las clases. ${ }^{6}$

Pero el nuevo alumno nativo digital tiene un enorme potencial. Es muy creativo (Palfrey y Gasser, 2016: 6-9), quiere decir algo diferente de los otros y, sobre todo, quiere hacer

\footnotetext{
${ }^{5}$ El director de Google, Laszlo Bock, en una entrevista al New York Times en junio de 2013, afirmaba: «Una de las cosas que hemos aprendido tras analizar todos los datos de nuestro proceso de selección es que el expediente académico y la puntuación de los candidatos en los test son inútiles como criterio de contratación».

${ }^{6}$ No se disponen todavía de datos estadísticos sobre el cambio de actitud del alumnado de los últimos años. Sin embargo, el debate social ha aumentado y los periódicos no cesan de dar noticias alarmantes relacionadas con el acoso escolar.
} 
las cosas de otra manera. Aprende de forma activa, le gusta manejar e interactuar. Es multitarea. Adora trabajar en equipo, le encanta buscar y descubrir en la red, para lo que posee habilidades técnicas extraordinarias (según los adultos -sean padres, abuelos o maestros- que se quedan pasmados ante la rapidez y pericia de los jóvenes, en relación a su dominio tecnológico pedestre).

Tampoco son asociales y solitarios. Y ni mucho menos incultos, como se dice por parte de muchos profesores y padres que afirman que la cultura general de los jóvenes es nula, ignorando que saben más de lo que se supone (Cassany y Ayala, 2008: 63).

\section{El nativo digital de $\mathrm{E} / \mathrm{LE}$}

Los profesores de idiomas y los de E/LE en concreto estamos de suerte. Ya que la comunicación es lo más importante en nuestra materia, es lógico que todos estos nuevos medios nos sean de gran utilidad. Sin embargo, siendo mejor que en otras asignaturas más teóricas, parece ser que también estas nuevas circunstancias con los primeros nativos digitales nos han pillado de sorpresa. Muchísimos profesores se limitan a seguir los manuales, que se reeditan cada año debido a una verdadera explosión editorial, pero que han cambiado poco (Andersson, 2011: 2) en metodología y contenidos en los últimos veinte años. Los ejercicios de gramática continúan siendo absolutos protagonistas de los cursos de E/LE. Los profesores, conscientes y obligados a hacer una enseñanza atractiva, se limitan a proponer al final de la clase algún juego o actividad lúdica (que hace resoplar de aburrimiento a los alumnos pues hacen los mismos juegos en todos los idiomas) o a poner una película, cortometraje o video de YouTube.

Pero el nuevo nativo digital no se conforma con eso. Así que surge la pregunta:

\section{1 ¿Cómo enseñar E/LE a estos nuevos nativos digitales?}

Hasta que se produzca el obligado cambio generacional, cuando los primeros nativos digitales se incorporen en las clases como docentes, el profesorado sigue siendo de la era analógica, es decir, profesores que aprendieron a enseñar en el siglo XX y que ahora están obligados a enseñar a alumnos del siglo XXI. Muchos de ellos son/fueron muy buenos profesores, muy bien documentados, incluso carismáticos, pero que ahora fracasan y no logran conectar con los alumnos de hoy. Curiosamente se da la paradoja de que los profesores más jóvenes, los que todavía no tienen 30 años, suelen ser incluso menos innovadores que sus colegas veteranos, ya que acaban de terminar estudios de grandes exigencias teóricas o han aprobado las oposiciones, sistema desfasado basado en la adquisición de conocimientos por la memoria y en acumulación de erudición, con nula o una mínima relación al mundo real. Por eso estos profesores jóvenes, muy próximos a sus alumnos por edad, suelen ser poco innovadores en sus clases, pues tampoco ellos han sido formados para el alumnado de hoy. En todo caso, no basta agregar algunos recursos técnicos en nuestros cursos para hacer metodología moderna y adaptada, basada en lo real y no solo en lo relevante, como decía Prensky (2011: 97). No es suficiente llevar todo el material en un lápiz de memoria, proyectar unos documentos word (a veces copiados de manuales) o un power point o poner un cortometraje de YouTube. Aunque es una buena forma de iniciar el camino a una 
enseñanza adaptada a nuestros alumnos de hoy.

\subsection{El modelo SAMR en E/LE}

El modelo SAMR es adecuado para aquellos profesores que, trabajando todavía con materiales analógicos, desean un acercamiento a las ventajas del material digital. El Doctor Rubén Puentedura (2009) fue el iniciador y posiblemente el que inventó el acrónimo SAMR (Substitution, Augmentation, Modification y Redefinition). Este modelo propone comenzar con actividades de sustitución pero sin hacer al principio un cambio integral de nuestros métodos. Poco a poco se va agregando tecnología con el fin de mejorar, modificar hasta redefinir totalmente la enseñanza, consiguiendo actividades más innovadoras.

En el caso del E/LE la fase de sustitución supondría lo que ya se hace con frecuencia, por ejemplo hacer ejercicios gramaticales en línea en lugar de dar fotocopias o proyectar un video o cortometraje de YouTube y dar una actividad. El aumento sería cuando, por ejemplo, enviamos la tarea por email y los alumnos la realizan en el ordenador añadiendo fotos u otros documentos multimedia. Ejemplos de modificación es hacer una tarea colaborativa en un google docs donde participan todos los alumnos, distribuyéndose el trabajo por equipos o individualmente, creando, por ejemplo, un espacio colaborativo en línea donde los estudiantes pueden retroalimentarse. Finalmente, la redefinición permite realizar actividades con ayuda de la técnica impensables hace apenas un par de años. Por ejemplo, hacer un proyecto colaborativo con todos los alumnos, filmarlo, hacer un video y subirlo a YouTube.

Esta es la práctica que se realizó en el Liceo Francés de Zurich, una actividad compleja, que se describirá a continuación.

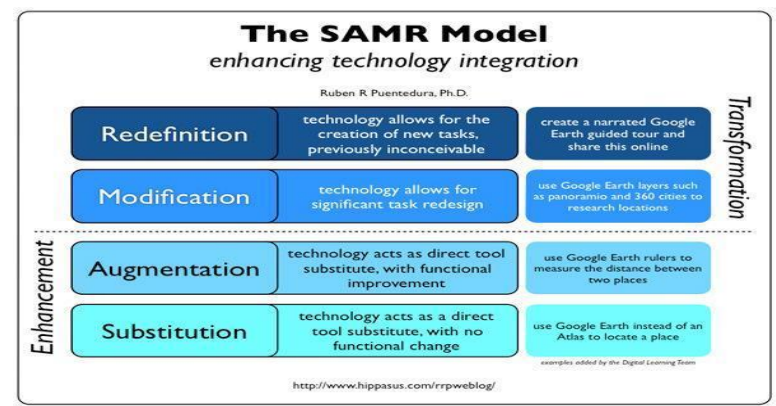

Imagen 1: Resumen Modelo SAMR de integración de TIC

\section{El proceso de creación de un telediario con el uso del Ipad}

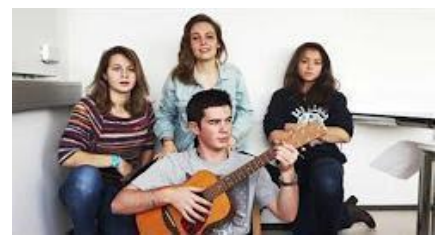

Imagen 2 extraida del vídeo LFZ-Journal Télévisé ${ }^{7}$

\footnotetext{
${ }^{7} \mathrm{https}: / /$ www.youtube.com/watch?v=Rsm6OVpw9Hk
} 
Este trabajo se hizo en el marco de un proyecto piloto de uso de los Ipads en la enseñanza del español LE en el Lycée Français de Zurich (a continuación LFZ) y tuvo su inicio en mayo de 2016. La nueva dirección del LFZ buscaba profesores voluntarios e implicados en las nuevas tecnologías para un pilotaje de enseñanza one to one (un alumno, un dispositivo) con los Ipads, con el objetivo de adaptar la escuela a los nuevos tiempos por medio de la innovación pedagógica, en caso de tener el proyecto buena acogida y de conseguir la financiación necesaria.

El principio fue muy agitado. El impacto en la enseñanza y en la dinámica de clase que tiene el dar a jóvenes adolescentes una tableta es enorme. Especialmente cuando esto ocurre por primera vez y sin un reglamento específico de utilización de los Ipads, sin filtros, donde los alumnos pueden descargar aplicaciones y visitar las páginas que deseen.

Sin querer minimizar el tema de la distracción que supone la tableta en clase, se observó que hay siempre un grupo de chicos que juega mucho, pero no son más de dos o tres por clase. Las chicas, por el contrario, no juegan, pero les encanta hacerse fotos. Observar todo esto y tratar de combatirlo fue irritante al principio, pero después de unos días, estas actividades cesaron casi por completo. No hay que olvidar que todos los jóvenes poseen un teléfono. No es de su interés proseguir estas actividades lúdicas en la escuela.

Más bien al contrario. Si se les propone algún proyecto interesante, se entregan a él con una creatividad y eficacia sorprendentes. Todos los estudiantes se implicaron con entusiasmo, también aquellos alumnos que normalmente no obtienen buenos resultados, pero que en este proyecto hicieron una aportación valiosa en cuestiones técnicas, por ejemplo, o con ideas originales.

El nuevo año escolar comenzó y pronto se pudieron incorporar las tabletas en un $80 \%$ del trabajo de curso. Adaptarse a la nueva dinámica de clase exigió algunos cambios. Por ejemplo, no se puede proponer una actividad completamente nueva en clases de más de 20 alumnos. El profesor es muy solicitado y el trabajo puede llegar a ser muy estresante para el profesor y frustrante para el alumno que tiene que esperar mucho para obtener una respuesta.

Por otro lado, las salas necesitan una disposición diferente, que permita la movilidad del profesor y los alumnos. Ideal es distribuir las mesas en pequeños grupos de 4 o 6 alumnos. Pero esta exigencia de orden práctico puede causar problemas en ciertos centros escolares.

En cuanto al comportamiento de los estudiantes fue mejorando una vez firmaron, ellos y sus padres, el reglamento de uso de los recursos informáticos ${ }^{8}$ y se integraron filtros. Por otro lado también observé la resistencia de algunos -pocos- alumnos que afirmaban que no les gustaba escribir en la tableta y que preferían las clases tradicionales.

Tras esta fase experimental donde pudimos conocer varias aplicaciones (quizlet, kahoot,

\footnotetext{
${ }^{8}$ Este es el reglamento (https://www.lfz.ch/fr/etablissement/code-de-conduite/charte-informatique.html) de uso de recursos informáticos y código de conducta en el LFZ, que firman los alumnos y los padres al inicio del curso. Hay numerosos ejemplos de Normativas de buenas prácticas y Reglamentos de uso de recursos informáticos en internet en español. Por ejemplo, el del colegio alba (file:///C:/Users/Usuario/Downloads/(http:/www.colegioalba.com/proyecto-ipad/)) en Madrid.
} 
BookCreator y muchas más) nos lanzamos a un proyecto más ambicioso, entre otras cosas motivada por la dirección que estaba organizando la inauguración del nuevo edificio del LFZ y que propuso a los profesores hacer exposiciones para que los visitantes conocieran más de cerca nuestro trabajo.

Así fue como surgió la idea de realizar un telediario del 26 de noviembre de 2016 con los estudiantes de segundo año de español. Desde el primer momento mostraron interés, incluso entusiasmo, por el proyecto.

\subsection{Las características del proyecto}

- Descripción: Enfoque por proyectos y orientado a la acción.

- Tarea final: Realización de un telediario del día de la inauguración del nuevo edificio del Liceo Francés de Zúrich (Suiza).

- Participantes: dos clases de 1ère (equivalente a $1^{\circ}$ de Bachillerato), 35 alumnos, segundo año de español, nivel B1, asignatura optativa.

- Duración: 3 semanas, a 3 horas por semana.

- Trabajo en equipos: 9 equipos que eligieron libremente los temas, así como la responsabilidad de cada miembro (presentadores, cámaras, montaje, etc).

- Objetivos: Práctica de la expresión oral. Tanto la formal como la comunicación espontánea (en el caso de las entrevistas) - Práctica de la expresión escrita (realización del guión y la traducción) - Trabajo en equipos, asumir responsabilidad por la tarea específica del grupo y por el trabajo final Actividad lúdica y gamificación, motivar a los alumnos a comunicar en español, darles a conocer la música y la cultura española de una forma divertida Aprendizaje transversal: de un proyecto así, aprende todo el mundo, los alumnos y la profesora.

- Destrezas: todas

- Competencias: todas

- Materiales empleados: Se ha realizado todo con la tableta (IPad) que se ha revelado como un excelente útil multifunción. Ipads para los estudiantes (one-toone); Mac Air para el montaje (IMovie), micrófonos de corbata.

- Herramientas: Pages o google docs para escribir el guión y compartir con la profesora para corregir. También para la traducción y creación del texto de subtitulado; cueprompter para entrenar y aprender el texto; la cámara del Ipad o Iphone para filmar; IMovie para hacer el montaje y escribir los subtítulos.

\subsection{EI desarrollo}

El desarrollo de la actividad se puede resumir de esta manera:

- Propuesta del tema.

- Formación de equipos (surgieron 9 grupos).

- Elección de actividad según el gusto o inclinación de cada equipo y asignación de roles (presentadores, reporteros, cámaras, montaje etc.).

- Escribir el guión por equipos. Se sube a un google docs colectivo. Corrección de la profesora

- Comienzan las pruebas para filmar. Ensayos de texto con cueprompter. Se busca el emplazamiento adecuado. Pruebas de sonido.

- Se hace la escaleta. Se escriben los textos de transición. Se ordenan las 
intervenciones.

- Selección y eliminación de escenas, cortes y montaje final.

- Cada grupo traduce y subtitula su intervención.

- Grabación de la escena final de baile con todos los participantes.

- Evaluación: Se evalúa en común entre la profesora y los alumnos. Hay una nota de clase, otra nota de equipo, donde por grupos tienen que negociar para distribuir los puntos según la aportación de cada miembro y una nota individual asignada por la profesora.

\subsection{Acogida del proyecto}

La respuesta por parte de los alumnos fue muy positiva. Intervinieron todos con gran motivación ( 35 alumnos en dos clases). Tuvo también muy buena acogida entre los padres, el equipo informático y la dirección de la escuela.

Dada la buena recepción de esta actividad, se presentó el video a un concurso y obtuvimos el Primer Premio a la "Mejor práctica docente de E/LE basada en el uso de las TIC" de la Editorial Difusión y Prolinter (Universidad Politécnica de Madrid) con la colaboración del Instituto Cervantes y el patrocinio de SIELE y FEDELE.

\subsection{Observaciones sobre la realización del telediario}

La forma de trabajar en una actividad como esta es muy diferente. Se permite la movilidad, cada grupo elige el mejor emplazamiento en total libertad. El éxito del trabajo depende de la implicación de los estudiantes, de su capacidad de organizarse entre ellos y de gestionar su autonomía. Por lo tanto, es indispensable una relación de confianza entre los alumnos y el profesor.

Esta forma de trabajo supone una actividad muy divertida y por lo tanto motivadora. Se estimula el sentimiento de cooperación, de apoyo mutuo y de solidaridad. Unos grupos que habían terminado, ayudaron a otros a finalizar su trabajo, sobre todo en actividades que eran nuevas para casi todos, incluido el profesor, como, por ejemplo, el subtitulado. Fue verdaderamente un trabajo colaborativo.

En general, el enfoque por proyectos traspasa el mero ejercicio académico. Los alumnos desarrollan competencias que después les serán muy útiles en la vida profesional.

\subsection{Observaciones sobre el trabajo con el Ipad}

En conclusión, trabajar en proyectos con el Ipad es pertinente e idóneo, ya que los alumnos de estas edades utilizan dispositivos móviles (smartphones, tabletas y portátiles) incesantemente y están muy familiarizados con internet. Por lo tanto, la receptividad de los estudiantes es muy satisfactoria. Concretamente el Ipad tiene muchas ventajas con respecto a otros ordenadores portátiles:

- Se inicia al momento y es rápido.

- Tiene una batería muy duradera.

- No hay virus y el mantenimiento informático es cómodo: ningún estudiante instalará nada peligroso.

- La portabilidad y el bajo peso. 
- Es más fácil organizar el trabajo en el Ipad que ordenar fotocopias y papeles. Puede, por lo tanto, impulsar la productividad y la organización, además de contribuir al desarrollo de competencias informáticas en los alumnos, algo que les ayudará posteriormente en el mundo profesional.

- La economía considerable de papel.

- La calidad de las presentaciones y de los trabajos realizados, lo que permite más creatividad y originalidad. La imagen y la comunicación cobran protagonismo en la enseñanza, algo a lo que los alumnos son muy receptivos.

- La colaboración (de los alumnos entre ellos y con el profesor).

- La posibilidad para el alumno de ir a su propio ritmo: el trabajo en autonomía y que se puede estudiar no importa dónde ni cuándo.

- La facilidad de anotar los documentos. Antes estaba prohibido escribir en el manual, ahora se anima al estudiante a hacerlo.

- Favorece el acceso a los manuales escolares, sobre todo los de última generación que están en línea.

\subsubsection{Los desafíos}

Cuando se plantea el uso del IPad en el aula surge una duda justificada: si supondrá una distracción para los alumnos. En general hay menos ruido en clase y hablan menos entre ellos.

- ¿Cuánto tiempo trabajan en clase y cuánto juegan? En una encuesta ${ }^{9}$ realizada en el LFZ al mes de comenzar el proyecto, la mayoría afirma haber trabajado entre el $50 \%$ y el $75 \%$ del tiempo.

- Es cierto que hay un grupo (bastante reducido, en general chicos) a los que les gusta mucho jugar. ¿Cómo afrontar esto? En primer lugar, con filtros potentes.

- Un problema supone la gestión de los trabajos de los alumnos, que puede llegar a ser extremadamente compleja, pues son múltiples las formas de entrega (por email, por AirDrop, compartiendo de nube a nube, etc). A veces es difícil localizar la ubicación de las tareas y llegar a controlar lo que los alumnos entregan y dónde se archiva.

- Desconocimiento de los recursos: por ejemplo, algunos profesores piensan que no hay nada en el Ipad para su materia.

- Hace falta tiempo para la formación de los profesores y la apropiación de los recursos por parte de estos.

\section{Conclusión}

Las ventajas relacionadas con la utilización del Ipad en clase superan con mucho los inconvenientes. El mayor desafío es, sin duda, el factor de distracción y seriedad en el trabajo, algo que mejorará con el uso generalizado de la tableta en la escuela.

\footnotetext{
${ }^{9}$ En el marco del proyecto piloto de enseñanza de E/LE con los Ipads, se realizó una encuesta a los alumnos (https://drive.google.com/file/d/0ByZ9EP_fVXvjYlZfRnAteXdBUTA/view?usp=sharing), a los padres (https://drive.google.com/file/d/0ByZ9EP_f fVXvjNkg0OHAzeUM0VEU/view? usp=sharing) у a los profesores (https://drive.google.com/file/d/0B3F9gmTqcYEFaTFqY1MxNEE3a1U/view?usp=sharing) en el LFZ al mes de comenzar el proyecto en junio de 2016. Fue llevada a cabo por la clase piloto y la profesora de español.
} 
¿Habrá en el futuro inmediato tabletas disponibles a todos los alumnos? Esa parece ser la tendencia y es lo que se desprende de un informe realizado en el marco de la Cumbre Mundial para la Innovación en la Educación en $2014^{10}$.

Estamos ante un gran desafío. Exigirá mucho de nosotros, los profesores, algo que ya nos llena de incertidumbre. Las dudas son comprensibles y los miedos justificados. En el peor de los escenarios imaginables, todos terminaremos siendo meros instructores técnicos. En el mejor, seremos actores necesarios de una nueva escuela, una escuela más justa, equitativa, menos reprobadora y más alegre, devolviendo al término escuela los valores de su etimología griega $\sigma \chi o \lambda \eta \dot{~ q u e ~ s i g n i f i c a ~ t r a n q u i l i d a d, ~ t i e m p o ~ l i b r e, ~ d o n d e ~ l o s ~}$ alumnos aprenden "haciendo", algo que no es nuevo", pues es la base de todas las corrientes pedagógicas más innovadoras de los últimos años.

\section{Bibliografía}

Andersson, P. (2011). La relevancia del material didáctico dentro del aula: Una investigación sobre las principales áreas de interés de los estudiantes de ELE. Recuperado de: portal.org/smash/record.jsf?pid=diva2\%3A519175\&dswid=-402

CASsANY, D. y Ayala, G. (2008). «Nativos e inmigrantes en la escuela, Investigaciones empíricas». Participación Educativa, 9, 53-71.

García, F., Portillo, J., Romo, J., Benito, M. (2007). Nativos digitales y modelos de aprendizaje. Recuperado de https://www.businessintelligence.info/assets/varios/nativos-digitales.pdf

Instituto Nacional DE Tecnologías Educativas Y DE Formación DEL PROFESORADO (INTEF) (2016). Diseñando el aula del futuro, Bring your own device (BYOD): una guía para directores y docentes. Recuperado de: http://blog.educalab.es/intef/wpcontent/uploads/sites/4/2016/02/Informe_resume n_BYOD_EUN_Enero_2016_INTEF.pdf

Moreno, A. (28.09.2015). «Así será la escuela en 2030». El Mundo. Recuperado de: http://www.elmundo.es/espana/2014/10/21/54455b9f22601d22738b458e.html.

OCDE (2015). Regards sur l'éducation 2015: Les indicateurs de l'OCDE. Recuperado de: http://www.keepeek.com/Digital-Asset-Management/oecd/

PAlfrey, J.G. y GASSER, Urs (2016). Born Digital: Understanding the First Generation of Digital Natives, New York: Basic Books.

Prensky, M. (2001). «Digital Natives, Digital Immigrants». On the Horizon, Vol. 9 No. 5., 1-6.

Prensky, M. ( 2011). Enseñar A Nativos Digitales. Madrid: Ediciones SM.

PUENTEDURA, R. (2014). Guiding development. Recuperado de: http://www.hippasus.com/rrpweblog/archives/2012/01/19/SAMR_GuidingDevel

\footnotetext{
${ }^{10}$ Véase nota 2

${ }^{11}$ Ya lo dijo Confucio: "Me lo contaron y lo olvidé; lo vi y lo entendí; lo hice y lo aprendí"
} 
opment.pdf

TEJEDOR, E. (24.09.2013) «Tener buenas notas ya no basta para conseguir un buen 